Review

\title{
Advances in Treatment of Post-Traumatic Stress Disorder with Chinese Medicine*
}

\author{
HU Guang-tao ${ }^{1}$ and WANG Yong ${ }^{2}$
}

\begin{abstract}
Timely, near, and expectation" is the main principle of battlefield rescue for military combat stress reaction (CSR). Post-traumatic stress disorder (PTSD) is the most common form of CSR and a long-term persistent mental disorder that is caused by unusual threatening or catastrophic psychological trauma. Chinese medicine (CM) has abundant resources, is simple, easy to master, with few side effects. This article summarizes the cellular and animal experimental mechanisms of CM treatment on PTSD, suggesting that traditional Chinese herbs and acupuncture can protect brain functional areas, and adjust hypothalamus-pituitary-adrenal axis. Traditional Chinese herbs and acupuncture have shown good anti-stress efficacy and fewer side effects in clinical application, which may improve the CSR in the battlefield.

KEYWORDS combat stress reaction, post-traumatic stress disorder, Chinese medicine, acupuncture treatment
\end{abstract}

As a special group, soldiers have high risk and high stress. The incidence of psychological problems and stress disorders is higher in the military groups than in the general population. ${ }^{(1)}$ Especially in the futuristic high-tech, information-based combat conditions and lurking unpredictable risk factors, the psychological impact of the combatants cannot be underestimated, which may lead to combat stress reaction (CSR), mainly manifesting as anxiety, depression, somatization disorder, compulsion, paranoia, insomnia and other symptoms. ${ }^{(2)}$ During the First World War, Salmond proposed three principles for dealing with wounded persons, namely, "timely, near, and expectation", in order to achieve 60\%-70\% rate of return of the wounded to the team, which significantly improved the combat effectiveness of the troops. ${ }^{(3)}$ At present, CSR reductions are properly managed, and more than $90 \%$ of the combat effectiveness can be restored. ${ }^{(4,5)}$

Post-traumatic stress disorder (PTSD) is the most common form of CSR. According to the speed or duration of the stress response, it can be divided into acute stress response, chronic stress response and PTSD. PTSD is more common in officers and soldiers who have experienced the trauma of war. ${ }^{(2,6)}$ Epidemiological surveys have shown that among people who have experienced traumatic events, the incidence of traumatic stress disorder varies from $50 \%-90 \%$, and the incidence of traumatic stress disorder is $5 \%-10 \% .^{(7,8)}$ An investigation conducted 4 years after the Yushu earthquake showed that the incidence of PTSD among local Tibetan adolescents was $19.3 \%$. ${ }^{\text {(9) }}$ With the advancement of research, the diagnostic criterion of PTSD has been continuously improved. The fifth edition of the "Statistical Diagnostic Handbook of Mental Disorders" published in 2013 described PTSD as a persistent mental and emotional disorder that appeared after a potentially lifethreatening event. The patient's main manifestations are pathological re-experiencing characterized by intrusive memory of traumatic events, avoidance of trauma-related cues, persistent hyper-arousal, choosing to forget the traumatic experience, and emotional numbing. ${ }^{(10)}$ During the 2019 coronavirus disease (COVID-19) pandemic, many infected patients and healthcare workers have shown psychiatric complications. Various factors were found to be

(C)The Chinese Journal of Integrated Traditional and Western Medicine Press and Springer-Verlag GmbH Germany, part of Springer Nature 2021

*Supported by Key Issues of the Logistics Support Department of the Chinese People's Liberation Army Military Commission (No. BLJ19J009) and Chongqing Science and Health Joint TCM Technology Key Project (No. ZY201801010)

1. Department of Psychological Medicine, Army 958th Hospital, Chongqing (400039), China; 2. Department of Traditional Chinese Medicine, Southwest Hospital, Army Medical University, Chongqing (400038), China

Correspondence to: Prof. WANG Yong, E-mail: wangyongjhy @ 126. com.

DOI: https://doi.org/10.1007/s11655-021-2864-1 
associated with higher risk of psychiatric symptoms and/or low psychological wellbeing, including women, people with poor self-related health, and relatives with COVID-19. ${ }^{(11)}$

A thorough search of the relevant literature showed no available published articles on the treatment of CSR with Chinese medicine (CM), although there are numerous published studies on the treatment of PTSD with CM. Therefore, we reviewed the mechanism and clinical research status of antagonism of PTSD by CM.

\section{PTSD Belongs to the Category of CM}

Although there is no clear record of the name of "PTSD" in CM, its clinical symptoms are well established. The disease belongs to the categories of CM, such as palpitations, insomnia, depression, epilepsy, madness, seizures, plum qi, forgetfulness, irritability, lily disease, benign qi, drowsiness, etc. ${ }^{(12)}$

Wei, et $\mathrm{al}^{(13)}$ believed that pathogenesis of PTSD in CM primarily involved Gan (Liver)-qi stagnation accompanied by phlegm obstruction. CM practitioners abroad are also actively exploring the use of existing literature, expert knowledge, and clinical diagnosis of CM to construct a PTSD diagnosis framework. The most likely pattern differentiation of PTSD was Xin (Heart)-Shen (Kidney) disorders caused by heat, fire, or a constitutional deficiency; Gan-qi stagnation; and Shen deficiency. ${ }^{(14)}$

\section{Research on Cellular and Animal Experimental Mechanisms of CM}

Before effective animal models and functional magnetic resonance imaging (MRI) techniques were applied to study PTSD, research on patients with PTSD mainly focused on peripheral bio-markers, including saliva, urine, blood pressure, and the concentrations of catecholamines and stress hormones. Among them, the high sensitivity of the norepinephrine system, the functional changes of the hypothalamus-pituitary-adrenal (HPA) axis, the highly sensitive glucocorticoid receptors, and the decline in circulating cortisol have been well established as reliable bio-markers that reflect the physiological and pathological changes of PTSD. ${ }^{(15-17)}$

With the emergence of emotional neuroscience and neuroimaging technologies, the direction of
PTSD research has also shifted from the periphery to specific neural circuits that affect the disease. Several physiological and pathological models based on neural circuits have gradually become well established, such as exaggerated threat detection model, abnormal fear learning model, diminished emotional regulation/ executive function and deficient context processing. ${ }^{(18)}$

\section{Protection of Brain Functional Areas}

The hippocampus is located in the temporal lobe of the brain and is part of the limbic system. The hippocampus is responsible for learning and memory in daily life, which involves conditional fear processing and the encoding process of declarative memory to the external environment. A study has shown that when body is stimulated by conditional fear, the inappropriate processing of signals by neurons can cause changes in the structure of the hippocampus, which in turn leads to changes in the interaction between the hippocampus and the amygdala. ${ }^{(19)}$ The function of the hippocampus is closely related to the amygdala. The amygdala and the hippocampus are sensitive parts of the central nervous system (CNS) stress response. Normal function of the amygdala is closely related to PTSD. ${ }^{(19)}$

$\mathrm{Hou}$, et $\mathrm{al}^{(20)}$ found that electroacupuncture at Baihui (DU 20) and Zusanli (ST 36) in rats could effectively regulate the protein expression level of neuronal nitric oxide synthase (NOS) in hippocampal cornu ammonis (CA) 1 and 3 regions, which in turn reduced nitric oxide release in the hippocampus, and may have a protective effect on hippocampal neurons. The elevated hippocampal neuronal NOS expression may be involved in the pathological process of PTSD. Electroacupuncture down-regulates the hippocampal neuronal NOS expression, which may be a potential mechanism for treatment of PTSD. Moreover, electroacupuncture can significantly improve the PTSDlike symptoms, up-regulate long-term potential in hippocampus, repair synaptic morphology and improve brain-derived neurotrophic factor (BDNF) levels in the amygdala and hippocampus. ${ }^{(21)}$ In addition, open field test and water maze test showed that gastrodin had anti-anxiety effect on PTSD animal model, and was related to interleukin-1 beta (IL-1 $\beta$ ) and IL- 6 in hippocampus, the expression of inducible NOS and the down-regulation of p38 phosphorylation status, suggesting that gastrodin may participate in the protection of hippocampal neurons through various 
mechanisms. ${ }^{(22)}$ A study showed that rosmarinic acid can alleviate the symptoms of PTSD animals by promoting hippocampal cell proliferation. This process was related to the up-regulation of extracellular regulated protein kinase expression. ${ }^{(23)}$

\section{Anti-stress and Adjustment of the HPA Axis}

The most notable feature of PTSD is the persistence of traumatic memory, easy activation, and persistent vigilance. Fear, learning and memory may be achieved through changes in synaptic efficacy.

Strong stimulation will lead to a series of neuroendocrine responses based on sympathetic nerve excitement, increase secretion of catecholamines and secretion of HPA cortex and improve the body's disease resistance. ${ }^{(24)}$ The activation of the HPA axis and the resultant increase in glucocorticosteroid secretion are the most important features of stress response. Traumatic events are the source of psychological stress in PTSD. Improving the stress status of PTSD patients may be an important research direction. ${ }^{(25)}$ Danzhi Xiaoyao Powder (丹桅逍遥散) is a famous CM prescription for treating Gan stagnation by soothing Gan and regulating qi. The chronic psychological stress model stimulated by uncertainty empty bottle accurately simulates anxiety of rats etiologically. Danzhi Xiaoyao Powder has a significant anti-anxiety effect. ${ }^{(26)}$ Xiaoyao Powder (逍遥散) has a great influence on the BDNF and tyrosine kinase $B$ (TrkB) of the stress state cortex and hippocampal CA1 and 3 regions, indicating a strong protective effect on the nervous system during stress; decreased BDNF in rat forehead cortex and hippocampal CA1 may participate in changes of chronic immobilization stress, which can be reversed by the Chinese herbs. The effect of Xiaoyaosan is better than that of Sijunzi Decoction (四君子汤) and Jingui Shenqi Pill (金质肾气 丸). ${ }^{(27)} \mathrm{Gao}$, et $\mathrm{al}^{(28)}$ established a type of social defeat stress mouse model to observe the efficacy and mechanism of Chaihu and Longgu Muli Decoction (柴 胡加龙骨牡蚛汤, TJ-12), and confirmed that doses of $1,0.5$ and $0.1 \mathrm{~g} / \mathrm{kg}$ could not only reduce the freezing percent scores of the contextual fear conditioning test, but also decrease the expression of BDNF gene in the hippocampus, indicating that TJ-12 may be effective for treating PTSD. There are relatively few studies on the improvement of stress status in PTSD patients by $\mathrm{CM}$, and new $\mathrm{CM}$ drugs for regulating stress status should be explored.
PTSD is an anxiety disorder associated with low levels of corticosterone and enhanced negative feedback of the HPA axis. The amygdala not only has an excitatory effect on the HPA axis, but also plays a key role in fear-related behaviors. Corticosterone exerts its actions through binding to the mineralocorticoid (MR) and glucocorticoid receptor (GR), which are abundant in the amygdala. ${ }^{(29)}$ The hippocampus is the integration site of stress response, and hippocampal GR/MR participates in the negative feedback regulation of HPA by affecting corticosterone. Single-prolonged stress (SPS) model is one of the animal models proposed for PTSD. SPS induces down-regulation of $M R$ and GR expression in the CA1 of hippocampus. SPS also induces significant decrease in MR and GR proteins in the hippocampus, although the degree of decreased expression of the receptors was different. In general, these findings suggested that MR and GR play critical roles in affecting hippocampal function. Changes in MR/GR ratio may be relevant for behavioral syndrome in PTSD. ${ }^{(29,30)}$ Different doses of Baihe Dihuang decoction (百合地黄汤) and fluoxetine can significantly down-regulate the expression of hippocampal GR and up-regulate the expression of MR in PTSD rats, which may be the mechanism of action. ${ }^{(31)}$ The ginsenoside $\mathrm{Rg} 1$ (5 mg/kg) treatment relieved PTSD-like behavior by altering elevated serum corticosterone and corticotrophin-releasing hormone $(\mathrm{CRH})$ levels in the hypothalamus. In contrast, fluoxetine $(3 \mathrm{mg} / \mathrm{kg}$ ) treatment reversed the behavioral changes, and had no effect on increased corticosterone and $\mathrm{CRH}$ levels. ${ }^{(32)}$ Notably, although repeated transcranial magnetic stimulation and electroacupuncture reduced anxiety in PTSD rats, electroacupuncture seems to have a more obvious effect in regulating HPA activity. ${ }^{(33)}$ Acupuncture can also regulate the abnormal expression of atrial natriuretic peptide and C-type natriuretic peptide, down-regulate corticosterone secretion and restore HPA activity to achieve anti-anxiety effect in a chronic stress anxiety rat model. ${ }^{(34)}$

\section{Clinical Applications of Herb Medicine in PTSD}

PTSD belongs to the category of emotional diseases in CM. Two important reasons for the occurrence of PTSD are panic and fear. ${ }^{(12)}$

Wei, et al $^{(35)}$ investigated the incidence of PTSD in the disaster victims and rescue forces of the 2008 Wenchuan earthquake, and observed the 
efficacy of Bailong Jieyu Granule (白龙解郁颗粒) on PTSD. The results showed that the incidence of PTSD reached $20.7 \%$ among the victims in 3 months after the earthquake, and was $3.1 \%$ among the rescue forces. After treatment with Bailong Jieyu Granule, the scores of somatization, compulsion, depression, anxiety, hostility, terror, and interpersonal sensitivity of PTSD victims decreased significantly compared with pre-treatment. In addition, a randomized, double-blind, placebo-controlled study of a Chinese herbal formula consisting of 14 ingredients (Xiaotan Jieyu Recipe, 消痰解郁方) was used to treat 245 survivors of the 2008 Sichuan earthquake. Compared with the placebo group, the PTSD-related symptoms of patients treated with this herbal formula were significantly improved. These herbs improved somatization, compulsive behavior, depression, anxiety, hostility and sleep quality without serious side effects, which indicated that Xiaotan Jieyu Recipe is a safe and effective option to improve the poor mental state of PTSD patients. ${ }^{(36)}$ Numata, et al ${ }^{(37)}$ reported that after the Great Eastern Japan earthquake and Tsunami, Kampo used Japanese traditional herbal medicine (Saikokokeishikankyoto, SKK) to treat PTSD. The efficacy of this therapy was studied in a randomized controlled trial (RCT), and the results showed that SKK significantly improved modified event scale score. Sub-scale analysis showed that compared with the control group, SKK significantly improved avoidance, over-excitement and aggressive behavior.

\section{Clinical Application of Acupuncture in PTSD}

Acupuncture has been mainly used to treat soft tissue injuries and bone and joint diseases caused by military training in the Chinese army. Acupuncture is widely used as a traditional therapy to treat mental disorders, including anxiety disorders and substance abuse. ${ }^{(38,39)}$ Although the exact mechanism of acupuncture remains unclear, increasing clinical evidence supports acupuncture for the treatment of various mental illnesses, including PTSD.

$\mathrm{Xu}$, et $\mathrm{al}^{(40)}$ used CM and acupuncture to interfere with depression caused by psychological stress. Both the $\mathrm{CM}$ and the acupuncture groups showed significantly lower scores of the depression selfrating scale and the anxiety self-rating scale. The acupuncture group showed significantly lower anxiety self-rating scale score as compared to the $\mathrm{CM}$ group $(P<0.05)$. Zhao, et $\mathrm{al}^{(41)}$ used acupuncture and brain waves to treat psychological stress response in military personnel, and used the Symptom Self-Rating Scale, the Beck Depression Scale and the Beck Anxiety Scale for random testing. The results showed that the scores of the three scales were significantly reduced after treatment $(P<0.05)$, and its efficacy was better than ordinary psychological counseling $(P<0.01)$. The 10 databases were searched to identify eligible RCTs. ${ }^{(42)}$ The random effects meta-analyses were performed to examined quality of the body of evidence (QoE) using the Grading of Recommendations Assessment, Development and Evaluation (GRADE). The authors identified very low QoE indicating that there is a significant difference in acupuncture treatment for PTSD symptoms (compared to any comparator) after intervention and patients with longer follow-up time for PTSD and depressive symptoms have lower QoE. The safety data indicated that the risk of serious adverse events is small, although some participants experienced minor/moderate pain, superficial bleeding, and hematoma at needle insertion sites.

A follow-up survey of 700,000 American soldiers who went to the Gulf War from 1990 to 1991 showed that nearly 100,000 soldiers had different types of cluster symptoms, including chronic fatigue, muscle fiber pain, irritable bowel syndrome, joint pain, depression, anxiety, and other mental symptoms. ${ }^{(43)}$ Extensive clinical and experimental data proved that acupuncture could effectively treat mental illnesses such as anxiety and depression, ${ }^{(44,45)}$ so the US military attempted to use acupuncture to treat PTSD. Wendling ${ }^{(46)}$ showed that acupuncture could improve the PTSD symptoms of veterans, and effectively reduce depression and pain compared to conventional treatment. Hollifield, et $\mathrm{al}^{(47)}$ randomly divided 84 patients diagnosed with PTSD into the acupuncture group (29 cases), the cognitive behavior therapy group (28 cases), and the control group (27 cases). The results showed that both acupuncture and cognitive behavior therapy could significantly improve depression and anxiety symptoms $(P<0.01)$. Conboy, et $\mathrm{al}^{(48)}$ randomized veterans with diagnosed symptoms of Gulf War Illness into either 6 months of biweekly acupuncture treatments (group 1, $n=52$ ) or 2 months of waitlist followed by weekly acupuncture treatments (group 2, $n=52$ ), and found that individualized acupuncture treatment of sufficient dose offered significant relief of physical disability and pain in these veterans. 
In the research on acupuncture treatment of PTSD, scalp acupuncture is the most extensive and effective. Clinical studies have shown that choosing scalp electroacupuncture in Baihui, Sishencong (EX-HN1), Shenting (DU 24), and Fengchi (GB 20) can regulate the glucose metabolism in the main brain areas of patients with PTSD, thereby achieving the purpose of treatment. ${ }^{(49)}$ If combined with moxibustion on Shenshu (BL 23), Mingmen (DU 4), Zhishi (BL 52), electroacupuncture is more effective than paroxetine in the treatment of PTSD, ${ }^{(50)}$ especially in improving anxiety symptoms.

A study to observe the effect of electroacupuncture at Baihui and Dazhui (Du 14) points in patients with PTSD and found that the scores of the electroacupuncture and Western medicine groups before, after, and 3 months and 6 months after treatment were generally similar, however, the levels of high alertness and SCL-90 somatization symptoms in the Essen Trauma questionnaire in the electroacupuncture group were lower than those in the Western medicine group. Electroacupuncture at Baihui and Dazhui points has similar overall efficacy as drug therapy in PTSD patients. Electroacupuncture at Baihui and Dazhui points was suggested to have similar overall efficacy as drug therapy in PTSD patients. Electroacupuncture showed better improvement of high alertness and somatization symptoms, with fewer side effects, good compliance, and low recurrence rate. ${ }^{(51)}$

Huang, et $\mathrm{al}^{(52)}$ used wrist-ankle acupuncture to treat PTSD. After 12 weeks, the therapeutic effect of wrist-ankle acupuncture was significantly better than the paroxetine control. Neiguan (PC 6), Changqiang (DU 1), and Shenmen (HT 7) points were also chosen in other studies. Acupuncture at these points had a definite effect on PTSD, and was more easily accepted than drug therapy. ${ }^{(53)}$ Acupuncture could be a useful tool for reducing pain and psychological symptoms related to earthquakes, but further research is required in this field. ${ }^{(54)}$

\section{Future Perspectives}

The pathogenesis of PTSD has not yet been fully elucidated. The therapeutic drugs against PTSD mainly target symptoms such as depression, epilepsy, and sedation. The US Food and Drug Administration has approved sertraline and paroxetine for the treatment of PTSD, but these drugs are expensive and have serious side effects. ${ }^{(55,56)}$ The research of CM for PTSD is still in its nascent stage, and its mechanism of action and metabolic pathway have not been fully clarified. It is necessary to further explore CM for the treatment of PTSD. In the absence of a precise treatment strategy, combination of CM, Western medicine and psychotherapy, as well as acupuncture, may be a better choice for the treatment of PTSD and may improve the therapeutic effect of CSR in the battlefield.

\section{Conflict of Interest}

The authors declare no competing interest.

\section{Author Contributions}

All the authors participated in generating the idea. Hu GT wrote the initial version of the manuscript. Wang $Y$ critically reviewed and submitted it.

\section{REFERENCES}

1. Gan LY, Meng SP, Feng ZZ, et al. The epidemiologic characteristic and risk factors of depression symptom in military personnel in high and cold regions. Chongqing Med (Chin) 2013;42:1332-1335.

2. Gray GC, Kaiser KS, Hawksworth AW, et al. Increased postwar symptoms and psychological morbidity among US Navy Gulf War veterans. Am J Trop Med Hyg 1999;60:758-766.

3. Friedman MJ, Schnurr PP, McDonagh-Coyle A. Posttraumatic stress disorder in the military veteran. Psychiatr Clin North Am 1994;17:265-277.

4. Gindi S, Galili G, Volovic-Shushan S, et al. Integrating occupational therapy in treating combat stress reaction within a military unit: An intervention model. Work 2016;55:737-745.

5. Svetlitzky V, Farchi M, Ben Yehuda A, et al. YaHaLOM training in the military: Assessing knowledge, confidence, and stigma. Psychol Serv 2020;17:151-159.

6. Baker MT, Moring JC, Hale WJ, et al. Acute assessment of traumatic brain injury and post-traumatic stress after exposure to a deployment-related explosive blast. Mil Med 2018;183:e555-e563.

7. Wittchen HU, Gloster A, Beesdo K, et al. Posttraumatic stress disorder: diagnostic and epidemiological perspectives. CNS Spectr 2009;14(1 Suppl 1):5-12.

8. Ren Z, Guo J, Yang C. Loss of homeland: a qualitative study of the changes in perception of relocated Sichuan earthquake survivors with posttraumatic stress disorder. BMC Psychiatry 2020;20:392.

9. Liu DL, Chen H, Ma L, et al. Post-traumatic stress disorder 
and its predictors among bereaved Tibetan adolescents four years after the Yushu earthquake: a cross-sectional survey in China. J Clin Nurs 2017;26:1095-1105.

10. American Psychiatric Association. Diagnostic and statistical manual of mental disorders. 5th ed. DSM-5. Philadedphia: 2013:4189.

11. Vindegaard N, Benros ME. COVID-19 pandemic and mental health consequences: Systematic review of the current evidence. Brain Behav Immun 2020;89:531-542.

12. Cai GX. Emotional diseases. Beijing: People's Medical Publishing House; 2011:134.

13. Wei PK, Shi J, Wu F, et al. Bailong Jieyu Granule in treatment of post-traumatic stress disorder among rescuing soldiers of Wenchuan Earth-quake. J Second Mil Med Univ (Chin) 2010;31:921-922.

14. Sinclair-Lian N, Hollifield M, Menache M, et al. Developing a traditional Chinese medicine diagnostic structure for post-traumatic stress disorder. J Altern Complement Med 2006;12:45-57.

15. Baker ME, Funder JW, Kattoula SR. Evolution of hormone selectivity in glucocorticoid and mineralocorticoid receptors. J Steroid Biochem Mol Biol 2013;137:57-70.

16. Knox D, Nault T, Henderson C, et al. Glucocorticoid receptors and extinction retention deficits in the single prolonged stress model. Neuroscience 2012;223:163-173.

17. Krisztina J Kovács. $\mathrm{CRH}$ : the link between hormonal metabolic-and behavioral responses to stress. J Chem Neuroanat 2013;54:25-33.

18. Liberzon I, Abelson JL. Context processing and the neurobiology of post-traumatic stress disorder. Neuron 2016;92:14-30.

19. Wilson MA, Grillo CA, Fadel JR, et al. Stress as a onearmed bandit: differential effects of stress paradigms on the morphology, neurochemistry and behavior in the rodent amygdala. Neurobiol Stress 2015;1:195-208.

20. Hou LQ, Liu S, Xiong KR. Effect of electroacupuncture on hippocampal nNOS expression in rats of post-traumatic stress disorder model. Chin Acupunct Moxibust (Chin) 2013;33:632-636.

21. Li M, Li K, Zhang $\mathrm{H}$, et al. Study on the mechanism of TMRK electroacupuncture in repairing synaptic plasticity in amygdala and hippocampus to relieve fear memory in PTSD rats. Technol Health Care 2019;27(S1):425-443.

22. Peng $Z$, Wang $H$, Zhang $R$, et al. Gastrodin ameliorates anxiety-like behaviors and IL-1 $\beta$ level and p38 MAPK phosphoylation of hippocampus in the rat model of posttraumatic stress disorder. Physiol Res 2013;62:537-545.

23. Nie $\mathrm{H}$, Peng $\mathrm{Z}$, Lao $\mathrm{N}$, et al. Rosmarinic acid ameliorates PTSD-like symptoms in a rat model and promotes cell proliferation in the hippocampus. Prog
Neuropsychopharmacol Biol Psychiatry 2014;51:16-22.

24. Herman JP, Cullinan WE. Neurocircuitry of stress: central control of the hypothalamo-pituitary adrenocortical axis. Trends Neurosci 1997;20:78-84.

25. Auxéméry Y. Post-traumatic psychiatric disorders: PTSD is not the only diagnosis. Presse Med 2018;47:423-430.

26. Li N, Tang QS, Zhao RZ, et al. Ethological changes in rats with chronic stress-induced anxiety and interventional effect of Danzhixiaoyao Powder. J Beijing Univ Tradit Chin Med (Chin) 2009;32:826-829.

27. Chen JX, Li Wei, Zhao X, et al. Effects of three Chinese formulas on BDNF, TrkB in rat contex and hippocampus with chronic immobilization stress. Chin J Pathophysiol (Chin) 2007;23:1296-1300.

28. Gao PF, Du YL, Xu YM, et al. Efficacy and mechanism of Chaihu and Longgu Muli Tang on posttraumatic stress disorder. Chin J Experi Tradit Med Formul (Chin) 2013;19:219-222.

29. Han F, Ding J, Shi Y. Expression of amygdala mineralocorticoid receptor and glucocorticoid receptor in the single-prolonged stress rats. BMC Neurosci 2014;15: 77.

30. Du Z, Han F, Shi YX. Expressions of hippocampal mineralocorticoid receptor (MR) and glucocorticoid receptor (GR) in the single-prolonged stress-rats. Acta Histochem Cytochem 2008;41:89-95.

31. Hu LL, Zhang YH, Su YG. Effects of Baihe Dihuang Decoction on behavior and GR/MR expression in hippocampus of rats with post traumatic stress disorder. Chin J Tradit Med Sci Technol (Chin) 2014;21:135-137.

32. Wang ZL, Zhu KX, Chen L, et al. Preventive effects of ginsenoside $\mathrm{Rg} 1$ on post-traumatic stress disorder (PTSD)-like behavior in male C57/B6 mice. Neurosci Lett 2015;605:24-28.

33. Fang YQ, Cai DJ, Zhou QZ, et al. Comparative research on intervention in anxiety behavior of PTSD-like rats by electroacupuncture and repetitive transcranial magnetic stimulation and serum corticosterone. J Nanjing Univ Tradit Chin Med (Natur Sci, Chin) 2012;28:357-359.

34. Yao H, Wei DN, Cai DJ, et al. Effects of acupuncture on ANP and CNP in adrenal gland and CORT in plasma in rats with chronic emotional stress anxiety. Chin Acupunct Moxibust 2016;36:169-174.

35. Wei PK, Xiu LJ, Pang B, et al. Effect of Bailong Jieyu Granule on the posttraumatic stress disorder due to earthquake. J Tradit Chin Med (Chin) 2010;5:510-512.

36. Meng XZ, Wu F, Wei PK, et al. A Chinese herbal formula to improve general psychological status in posttraumatic stress disorder: A randomized placebo-controlled trial on Sichuan arthquake survivors. Evid Based Complement Altern Med 2012;2012:691258.

37. Numata T, Gunfan S, Takayama S, et al. Treatment of posttraumatic stress disorder using the traditional Japanese 
herbal medicine saikokeishikankyoto: A randomized, observer-blinded, controlled trial in survivors of the Great East Japan earthquake and tsunami. Evid Based Complement Altern Med 2014; 2014:683293.

38. Lin JG, Chan YY, Chen YH. Acupuncture for the treatment of opiate addiction. Evid Based Complement Altern Med 2012;2012:739045.

39. Rometsch-Ogioun El, Sount C, Windthorst $P$, et al. Chronic pain in refugees with posttraumatic stress disorder (PTSD): A systematic review on patients' characteristics and specific interventions. J Psychosom Res 2019;118:83-97.

40. Xu JY, Wang FQ, Liu QA, et al. The influence of traditional Chinese medicine and acupuncture on SDS and SAS of anti-terrorism exercise personnel. Med Chin People's Armed Police Forces (Chin) 2004;15:940-941.

41. Zhao MJ, Zhang JT, Guo HY, et al. Analysis of 33 cases of military mental stress response intervened by acupuncture and brain wave therapy. Med J Chin People's Liberation Army (Chin) 2011;36:982.

42. Grant S, Colaiaco B, Motala A, et al. Acupuncture for the treatment of adults with posttraumatic stress disorder: A systematic review and meta-analysis. J Trauma Dissociation 2018;19:39-58.

43. Ozakinci G, Hallman WK, Kipen HM. Persistence of symptoms in veterans of the First Gulf War: 5-year followup. Environ Health Perspect 2006;114:1553-1537.

44. Karen P. Anxiety, depression and acupuncture: A review of the clinical research. Auton Neurosci 2010;157:91-95.

45. Asher GN, Gerkin J, Gaynes BN. Complementary therapies for mental health disorders. Med Clin North Am 2017;101:847-864.

46. Wendling P. Acupuncture lessens PTSD, depression, pain in veterans [N/OL]. (2008-12-15) [11] [2013-05-04]. http:// psych.imng.com/filead-min/content_pdf/imn/archive_pdf/ vol4iss24/71340_main.pdf.

47. Hollifield M, Sinclair-Lian N, Warner T D, et al. Acupuncture for posttraumatic stress disorder: a randomized controlled pilot trial. J Nerv Ment Dis 2007;195:504-513.

48. Conboy L, Gerke T, Hsu KY, et al. The effectiveness of individualized acupuncture protocols in the treatment of Gulf War Illness: A pragmatic randomized clinical trial. PLoS One 2016;11:e0149161.

49. Zhang $H$, Chen WW, Song WZ, et al. Brain glucose metabolism involved in acupuncture treatment on posttraumatic stress disorder. Chin J Tradit Chin Med Pharm (Chin) 2010;25:1882-1884.

50. Zhang H, Yuan CF, Ran LH, et al. RCT research of different acupuncture therapies in treating posttraumatic stress disorder after Wenchuan'5.12'earthquake. Chin J Tradit Chin Med Pharm (Chin) 2010; 25:1505-1510.

51. Zhao GJ, Liu MY, Guo SY, et al. Observation on therapeutic effect of electroacupuncture at Baihui-Dazhui point on posttraumatic stress disorder. J Clin Acupunct Moxibust (Chin) 2014;30:31-33.

52. Huang LJ, Luo WJ, Zhu H, et al. Effect of wrist-ankle acupuncture on post traumatic stress disorder after work-related injury. Chin J Rehabilit Theory Pract (Chin) 2012;18:855-857.

53. Hong C, Efferth T. Systematic review on post-traumatic stress disorder among survivors of the Wenchuan earthquake. Trauma Violence Abuse 2016;17:542-561.

54. Moiraghi C, Poli P, Piscitelli A. An observational study on acupuncture for earthquake-related post-traumatic stress disorder: The experience of the lombard association of medical acupuncturists/acupuncture in the world, in Amatrice, Central Italy. Med Acupunct 2019;31:116-122.

55. Lochmann D, Richardson T. Selective serotonin reuptake inhibitors. Handb Exp Pharmacol 2019;250:135-144.

56. Woroń J, Siwek M, Gorostowicz A. Adverse effects of interactions between antidepressants and medications used in treatment of cardiovascular disorders. Psychiatr Pol 2019;53:977-995.

(Accepted September 28, 2020; First Online May 31, 2021) Edited by ZHANG Wen 